PROCEEDINGS OF THE

AMERICAN MATHEMATICAL SOCIETY

Volume 133, Number 9, Pages 2613-2620

S 0002-9939(05)08052-4

Article electronically published on April 19, 2005

\title{
DECAY AND GROWTH FOR A NONLINEAR PARABOLIC DIFFERENCE EQUATION
}

\author{
SERGIU HART AND BENJAMIN WEISS
}

(Communicated by David S. Tartakoff)

\begin{abstract}
We prove a difference equation analogue of the decay-of-mass result for the nonlinear parabolic equation $u_{t}=\Delta u+\mu|\nabla u|$ when $\mu<0$, and a new growth result when $\mu>0$.
\end{abstract}

\section{INTRODUCTION}

Consider the following difference equation:

$$
\begin{aligned}
u_{i}^{n+1}-u_{i}^{n}= & \alpha\left(u_{i+1}^{n}-2 u_{i}^{n}+u_{i-1}^{n}\right) \\
& +\mu\left(\left|u_{i}^{n}-u_{i-1}^{n}\right|+\left|u_{i}^{n}-u_{i+1}^{n}\right|\right), i \in \mathbb{Z}, n \in \mathbb{Z}_{+},
\end{aligned}
$$

starting with some $u^{0}=\left(u_{i}^{0}\right)_{i \in \mathbb{Z}}$ such that $u^{0} \geq 0$ and $\sum_{i \in \mathbb{Z}} u_{i}^{0}<\infty$, where the parameters $\mu$ and $\alpha$ satisfy

$$
0<|\mu| \leq \alpha \text { and } \alpha+|\mu| \leq \frac{1}{2}
$$

This scheme corresponds (after appropriate rescaling) to the following partial differential equation for $u(x, t)$ :

$$
u_{t}=u_{x x}+\mu\left|u_{x}\right|, x \in \mathbb{R}, t \in \mathbb{R}_{+},
$$

with initial condition $u(x, 0)=u^{0}(x)$ such that $u^{0} \geq 0$ and $\int_{\mathbb{R}} u^{0}(x) \mathrm{d} x<\infty$ (as usual, $u_{i}^{n}$ in (11) corresponds to $\left.u(i \Delta x, n \Delta t)\right)$. The behavior of the total mass $\int_{\mathbb{R}} u(x, t) \mathrm{d} x$ as $t \rightarrow \infty$ is as follows:

(D) When $\mu<0$ the mass decays to zero: $\int_{\mathbb{R}} u(x, t) \mathrm{d} x \rightarrow 0$ as $t \rightarrow \infty$; see Ben-Artzi, Goodman and Levy [1, Theorem 5.1].

(G) When $\mu>0$ the mass grows to infinity: $\int_{\mathbb{R}} u(x, t) \mathrm{d} x \rightarrow \infty$ as $t \rightarrow \infty$ (for $\left.u^{0} \neq 0\right)$; see Laurençot and Souplet [4, Theorem 1(i)].

Here we prove, first, that the difference equation (1) satisfies a decay-of-mass result that is analogous to (D); and second, that it satisfies a growth result stronger than $(\mathrm{G})$ :

$(\boldsymbol{\Delta})$ When $\mu<0$ the mass decays to zero: $\sum_{i \in \mathbb{Z}} u_{i}^{n} \rightarrow 0$ as $n \rightarrow \infty$; see Theorem 3.

( $\boldsymbol{\Gamma})$ When $\mu>0$ there is convergence to a constant: for each $u^{0} \neq 0$ there is a constant $c>0$ such that $\lim _{n \rightarrow \infty} u_{i}^{n}=c$ for all $i$; see Theorem 6 .

Received by the editors January 28, 2004 and, in revised form, March 27, 2004.

2000 Mathematics Subject Classification. Primary 35K15, 35K55, 39A05; Secondary 60J10.

(C)2005 American Mathematical Society 
Moreover, the result $(\Gamma)$ applies to any bounded (not necessarily summable) initial condition $u^{0}$. Finally, both results $(\Delta)$ and $(\Gamma)$ (like $(D)$ and $(G)$ ) extend to the multi-dimensional case; see Theorems 5 and 8

We would like to acknowledge useful discussions with Matania Ben-Artzi, who presented the discrete decay-of-mass problem, and with Eli Shamir, with whom that question originated. We thank Philippe Laurençot for noting an error in an earlier version of this paper, and we thank the referee for his careful reading and useful comments, and for pointing out that, in the PDE setup, the preprint of Gilding, Guedda and Kersner [3, Theorems 11 and 16] provides a strengthening of $(\mathrm{G})$, which parallels our $(\Gamma)$.

\section{Preliminaries}

Let $\ell^{\infty}(\mathbb{Z})=\left\{u=\left(u_{i}\right)_{i \in \mathbb{Z}}: \sup _{i \in \mathbb{Z}}\left|u_{i}\right|<\infty\right\}$ be the space of doubly infinite bounded sequences, and let $\ell^{1}(\mathbb{Z})=\left\{u=\left(u_{i}\right)_{i \in \mathbb{Z}}:\|u\|<\infty\right\}$ be the subspace of summable sequences, where $\|\cdot\|$ denotes the $\ell^{1}$-norm $\|u\|=\sum_{i \in \mathbb{Z}}\left|u_{i}\right|$. Put $\ell_{+}^{\infty}(\mathbb{Z})=\left\{u \in \ell^{\infty}(\mathbb{Z}): u \geq 0\right\}$ (all inequalities $u \geq v$ are meant coordinatewise: $u_{i} \geq v_{i}$ for all $i$ ); similarly for $\ell_{+}^{1}(\mathbb{Z})$.

Given parameters $\mu$ and $\alpha$ that satisfy (2), define $F: \ell_{+}^{\infty}(\mathbb{Z}) \rightarrow \ell_{+}^{\infty}(\mathbb{Z})$ by

$$
\begin{aligned}
F_{i}(u):= & (1-2 \alpha) u_{i}+\alpha\left(u_{i-1}+u_{i+1}\right) \\
& +\mu\left(\left|u_{i}-u_{i-1}\right|+\left|u_{i}-u_{i+1}\right|\right)
\end{aligned}
$$

for each $i \in \mathbb{Z}$, and $F(u)=\left(F_{i}(u)\right)_{i \in \mathbb{Z}}$. The conditions on $\mu$ and $\alpha$ guarantee that indeed $F(u) \in \ell_{+}^{\infty}(\mathbb{Z})$ when $u \in \ell_{+}^{\infty}(\mathbb{Z})$; moreover, $F(u) \in \ell_{+}^{1}(\mathbb{Z})$ when $u \in$ $\ell_{+}^{1}(\mathbb{Z})$ (see Lemma 1 below). We write $F^{(n)}(u)$ for the $n$-th iterate of $F$, i.e., $F^{(1)}(u)=F(u)$ and $F^{(n)}(u)=F\left(F^{(n-1)}(u)\right)$. Then (1) is just $u^{n+1}=F\left(u^{n}\right)$, and so $u^{n}=F^{(n)}\left(u^{0}\right)$.

Lemma 1. F satisfies:

(i) $F(u) \in \ell_{+}^{\infty}(\mathbb{Z})$ for all $u \in \ell_{+}^{\infty}(\mathbb{Z})$.

(ii) $F(u) \in \ell_{+}^{1}(\mathbb{Z})$ for all $u \in \ell_{+}^{1}(\mathbb{Z})$.

(iii) $\|F(u)\| \leq\|u\|$ when $\mu<0$, and $\|F(u)\| \geq\|u\|$ when $\mu>0$, for all $u \in \ell_{+}^{1}(\mathbb{Z})$.

(iv) $F$ is monotonic: $F(u) \leq F(v)$ for all $u, v \in \ell_{+}^{\infty}(\mathbb{Z})$ with $u \leq v$.

Proof. $F_{i}(u)$ is a convex combination of $u_{i-1}, u_{i}, u_{i+1}$ (the coefficients are among $\alpha \pm \mu, 1-2 \alpha \pm 2 \mu$, and $1-2 \alpha$, which are all nonnegative by (2)), which proves (i). When $u \in \ell_{+}^{1}(\mathbb{Z})$, we have $\sum_{i} F_{i}(u)=\sum_{i} u_{i}+2 \mu \sum_{i}\left|u_{i}-u_{i-1}\right| \leq(1+4|\mu|) \sum_{i} u_{i}<$ $\infty$, which proves (ii) and (iii). For (iv), $F_{i}(u)$ is a continuous piecewise linear function of $u_{i-1}, u_{i}, u_{i+1}$ (there are four regions, determined by the signs of $u_{i}-u_{i-1}$ and $u_{i}-u_{i+1}$ ). In each region $F_{i}(u)$ is monotonic (it is a convex combination of its arguments), and the continuous "gluing" of these pieces is therefore also monotonic. More precisely, given $u \leq v$, one can find a chain $u=v^{0} \leq v^{1} \leq \ldots \leq v^{m}$ such that $v^{k-1}$ and $v^{k}$ belong to the same region of linearity of $F_{i}$ for each $k=1, \ldots, m$, and the endpoint $v^{m}$ satisfies $v_{j}^{m}=v_{j}$ for $j=i-1, i, i+1$ (indeed: increase in turn each one of the three coordinates $j=i-1, i, i+1$ starting from $u_{j}$, until either the boundary of a region is crossed - this happens when $w_{i}=w_{i-1}$ or $w_{i}=w_{i+1}$ or $v_{j}$ is reached). Thus $F_{i}\left(v^{k-1}\right) \leq F_{i}\left(v^{k}\right)$ (the two points are in the same region) for all $k=1, \ldots, m$, and $F_{i}\left(v^{m}\right)=F_{i}(v)$, which completes the proof. 
We introduce an auxiliary operator $G: \ell_{+}^{\infty}(\mathbb{Z}) \rightarrow \ell_{+}^{\infty}(\mathbb{Z})$ defined by

$$
G_{i}(u):= \begin{cases}(\alpha+\mu) u_{i-1}+(1-2 \alpha) u_{i}+(\alpha-\mu) u_{i+1}, & \text { for } i \geq 1, \\ (\alpha-\mu) u_{-1}+(1-2 \alpha+2 \mu) u_{0}+(\alpha-\mu) u_{1}, & \text { for } i=0 \\ (\alpha-\mu) u_{i-1}+(1-2 \alpha) u_{i}+(\alpha+\mu) u_{i+1}, & \text { for } i \leq-1\end{cases}
$$

and $G(u)=\left(G_{i}(u)\right)_{i \in \mathbb{Z}}$. Thus $G(u)$ is obtained from $F(u)$ when each term $\left|u_{j}-u_{j+1}\right|$ is replaced by $u_{j}-u_{j+1}$ for $j \geq 0$, and by $u_{j+1}-u_{j}$ for $j \leq-1$. Note that $F(u)=G(u)$ whenever $u$ is unimodal with mode at 0 ("centered unimodal"), i.e., $u_{i} \geq u_{i+1}$ for $i \geq 0$ and $u_{i} \geq u_{i-1}$ for $i \leq 0$.

Lemma 2. G satisfies:

(i) $G$ is a linear monotonic operator.

(ii) $\|G(u)\| \leq\|u\|$ when $\mu<0$, and $\|G(u)\| \geq\|u\|$ when $\mu>0$, for all $u \in \ell_{+}^{1}(\mathbb{Z})$.

(iii) $F(u) \leq G(u)$ when $\mu<0$, and $F(u) \geq G(u)$ when $\mu>0$, for all $u \in \ell_{+}^{\infty}(\mathbb{Z})$.

(iv) $F^{(n)}(u) \leq G^{(n)}(u)$ when $\mu<0$, and $F^{(n)}(u) \geq G^{(n)}(u)$ when $\mu>0$, for all $u \in \ell_{+}^{\infty}(\mathbb{Z})$ and all $n \geq 1$.

Proof. (i) is immediate. (ii) follows from $\|G(u)\|=\|u\|+4 \mu u_{0}$. For (iii), let $i \geq 1$; we have

$$
\begin{aligned}
\frac{1}{\mu}\left(F_{i}(u)-G_{i}(u)\right)= & \left|u_{i}-u_{i-1}\right|+\left|u_{i}-u_{i+1}\right| \\
& -\left(u_{i}-u_{i-1}\right)-\left(u_{i+1}-u_{i}\right) \geq 0,
\end{aligned}
$$

so $F_{i}(u) \leq G_{i}(u)$ when $\mu<0$, and $F_{i}(u) \geq G_{i}(u)$ when $\mu>0$; similarly when $i \leq-1$ and $i=0$. Finally, (iv) follows by induction on $n$ : when $\mu<0$, from $F^{(n)}(u) \leq G^{(n)}(u)$ and the monotonicity of $G$ follows $G\left(F^{(n)}(u)\right) \leq G\left(G^{(n)}(u)\right)$, and from (iii) follows $F\left(F^{(n)}(u)\right) \leq G\left(F^{(n)}(u)\right)$, which together yield $F^{(n+1)}(u) \leq$ $G^{(n+1)}(u)$; similarly when $\mu>0$.

\section{DECAY OF MASS}

We now assume that $\mu<0$; put $\lambda=|\mu|$. Lemma 1)(iii) implies that the total mass $\sum_{i} u_{i}^{n}$ decreases with $n$; the result below shows that in fact it decays to zero.

Theorem 3. Let $\mu<0$ and $\alpha$ satisfy (2). Then for all $u^{0} \in \ell_{+}^{1}(\mathbb{Z})$

$$
\lim _{n \rightarrow \infty} \sum_{i \in \mathbb{Z}} u_{i}^{n}=0
$$

To prove the theorem we will show that $\left\|G^{(n)}\left(u^{0}\right)\right\| \rightarrow_{n} 0$ and then use Lemma 2 (iv). Take $q=\alpha /(\alpha+\lambda)$, and let $z=\left(q^{|i|}\right)_{i \in \mathbb{Z}} \in \ell_{+}^{1}(\mathbb{Z})$.

Lemma 4. There exists $0<\rho<1$ such that $G(z) \leq(1-\rho) z$.

Proof. For $i \geq 1$ we have

$$
\begin{aligned}
G_{i}(z) & =(\alpha-\lambda) q^{i-1}+(1-2 \alpha) q^{i}+(\alpha+\lambda) q^{i+1} \\
& =\left(1-\frac{\lambda^{2}}{\alpha}\right) q^{i}
\end{aligned}
$$

(recall that $q=\alpha /(\alpha+\lambda)$ ). Similarly for $i \leq-1$. Finally, for $i=0$,

$$
G_{0}(z)=(1-2 \alpha-2 \lambda)+2(\alpha+\lambda) q<1-\frac{\lambda^{2}}{\alpha} \text {. }
$$

Take $\rho=\lambda^{2} / \alpha$. 
There is nothing special about this value of $q$; we choose it for convenience only (any $q$ close enough to 1 , specifically $(\alpha-\lambda) /(\alpha+\lambda)<q<1$, will do). Also, note that $F(z)=G(z)$ since $z$ is centered unimodal.

Proof of Theorem 3. Let $q, z$ and $\rho$ be as above. Given $u \in \ell_{+}^{1}(\mathbb{Z})$, for each $k \geq 0$ let $v^{[k]} \in \ell_{+}^{1}(\mathbb{Z})$ be the $k$-truncation of $u$, i.e., $v_{i}^{[k]}:=u_{i}$ for $i=-k, \ldots, k$ and $v_{i}^{[k]}:=0$ otherwise, and define $\theta_{k}:=\max _{i=-k, \ldots, k} u_{i} / q^{|i|}$. Then $v^{[k]} \rightarrow_{k} u$ and $v^{[k]} \leq \theta_{k} z$. By Lemmata 2(i) and4 (iterated $n$ times), we get

$$
G^{(n)}\left(v^{[k]}\right) \leq G^{(n)}\left(\theta_{k} z\right)=\theta_{k} G^{(n)}(z) \leq \theta_{k}(1-\rho)^{n} z .
$$

Also, $\left\|G^{(n)}\left(u-v^{[k]}\right)\right\| \leq\left\|u-v^{[k]}\right\|$ by Lemma 2(ii). Therefore

$$
\begin{aligned}
\left\|G^{(n)}(u)\right\| & =\left\|G^{(n)}\left(v^{[k]}\right)\right\|+\left\|G^{(n)}\left(u-v^{[k]}\right)\right\| \\
& \leq \theta_{k}(1-\rho)^{n}\|z\|+\left\|u-v^{[k]}\right\| .
\end{aligned}
$$

But $0<1-\rho<1$, so $\lim \sup _{n \rightarrow \infty}\left\|G^{(n)}(u)\right\| \leq\left\|u-v^{[k]}\right\|$. This holds for all $k$, which together with $\left\|u-v^{[k]}\right\| \rightarrow 0$ as $k \rightarrow \infty$ shows that $\left\|G^{(n)}(u)\right\| \rightarrow 0$ as $n \rightarrow \infty$; recalling that $0 \leq F^{(n)}(u) \leq G^{(n)}(u)$ by Lemma 2 (iv) completes the proof.

\section{DeCAy In higher Dimensions}

Let $d \geq 1$ be an integer. The $d$-dimensional version of (3) is the differential equation

$$
u_{t}=\Delta u+\mu|\nabla u|, x \in \mathbb{R}^{d}, t \in \mathbb{R}_{+} .
$$

The decay-of-mass result of Ben-Artzi, Goodman and Levy [1, Theorem 5.1] for this equation, when $\mu<0$, holds for any dimension $d$. Our result of Theorem 3 also generalizes to $d$ dimensions.

Let $\mathbb{Z}^{d}$, the space of $d$-dimensional integer vectors $i=\left(i_{1}, \ldots, i_{d}\right)$, be endowed with the $\ell^{1}$-norm $\|i\|=\sum_{r=1}^{d}\left|i_{r}\right|$, and put $\ell^{\infty}\left(\mathbb{Z}^{d}\right)=\left\{u=\left(u_{i}\right)_{i \in \mathbb{Z}^{d}}: \sup _{i \in \mathbb{Z}^{d}}\left|u_{i}\right|<\infty\right\}$ and $\ell^{1}\left(\mathbb{Z}^{d}\right)=\left\{u=\left(u_{i}\right)_{i \in \mathbb{Z}^{d}}:\|u\|<\infty\right\}$, where $\|u\|=\sum_{i \in \mathbb{Z}^{d}}\left|u_{i}\right|$. Given $\mu$ and $\alpha$ such that

$$
0<|\mu| \leq \alpha \text { and } \alpha+|\mu| \leq \frac{1}{2 d},
$$

define $F: \ell_{+}^{\infty}\left(\mathbb{Z}^{d}\right) \rightarrow \ell_{+}^{\infty}\left(\mathbb{Z}^{d}\right)$ by $F(u)=\left(F_{i}(u)\right)_{i \in \mathbb{Z}^{d}}$ and

$$
F_{i}(u):=(1-2 d \alpha) u_{i}+\alpha \sum_{j \in V(i)} u_{j}+\mu \sum_{j \in V(i)}\left|u_{i}-u_{j}\right|
$$

for each $i \in \mathbb{Z}^{d}$, where $V(i):=\left\{j \in \mathbb{Z}^{d}:\|j-i\|=1\right\}$ denotes the 1-neighborhood of $i$ (i.e., those $j$ that are obtained from $i$ by increasing or decreasing one coordinate by 1$)$. Put $u^{n}:=F^{(n)}\left(u^{0}\right)$.

To define the auxiliary operator $G$, for each $i \in \mathbb{Z}^{d}$ we partition $V(i)$ into $V_{+}(i):=$ $\left\{j \in \mathbb{Z}^{d}:\|j\|=\|i\|+1\right\}$ and $V_{-}(i):=\left\{j \in \mathbb{Z}^{d}:\|j\|=\|i\|-1\right\}$, and put

$$
\begin{aligned}
G_{i}(u):= & (1-2 d \alpha) u_{i}+\alpha \sum_{j \in V(i)} u_{j} \\
& +\mu \sum_{j \in V_{+}(i)}\left(u_{i}-u_{j}\right)+\mu \sum_{j \in V_{-}(i)}\left(u_{j}-u_{i}\right) .
\end{aligned}
$$


This can be rewritten as

$$
\begin{aligned}
G_{i}(u)= & \left(1-2 d \alpha+\left[\left|V_{+}(i)\right|-\left|V_{-}(i)\right|\right] \mu\right) u_{i} \\
& +(\alpha+\mu) \sum_{j \in V_{-}(i)} u_{j}+(\alpha-\mu) \sum_{j \in V_{+}(i)} u_{j},
\end{aligned}
$$

where $|A|$ denotes the number of elements of a finite set $A$ (compare with (4)).

It is straightforward to check that Lemmata 1 and 2 continue to hold. As for Lemma 4 (for $\lambda=-\mu>0$ ), we again take $q=\alpha /(\alpha+\lambda)$ and put $z=\left(q^{\|i\|}\right)_{i \in \mathbb{Z}^{d}} \in$ $\ell_{+}^{1}\left(\mathbb{Z}^{d}\right)$. The set $V_{+}(i)$ contains $d+m$ elements, where $m$ is the number of coordinates of $i$ that vanish. Increasing $z_{j}$ from $q^{\|i\|+1}$ to $q^{\|i\|-1}$ for $m$ of the elements $j$ of $V_{+}(i)$ can only increase $G_{i}(z)$; hence

$$
\begin{aligned}
G_{i}(z) & \leq(1-2 d \alpha) q^{\|i\|}+d(\alpha-\lambda) q^{\|i\|-1}+d(\alpha+\lambda) q^{\|i\|+1} \\
& =\left(1-\frac{d \lambda^{2}}{\alpha}\right) q^{\|i\|}=(1-\rho) z_{i} .
\end{aligned}
$$

Therefore the proof of Theorem 3 in the previous section applies to the $d$-dimensional case as well (with the appropriate trivial adjustments, like $\|i\| \leq k$ instead of $i=-k, \ldots, k)$. Thus we have

Theorem 5. Let $d \geq 1$ be an integer, and let $\mu<0$ and $\alpha$ satisfy (51). Then for all $u^{0} \in \ell_{+}^{1}\left(\mathbb{Z}^{d}\right)$

$$
\lim _{n \rightarrow \infty} \sum_{i \in \mathbb{Z}^{d}} u_{i}^{n}=0
$$

\section{Growth}

We now return to the one-dimensional case and assume that $\mu>0$. Here the total mass $\sum_{i} u_{i}^{n}$ increases (recall Lemma1(iii)), and we will show that $u^{n}$ always converges to a constant sequence $(\ldots, c, c, c, \ldots)$ for some $c>0$. In fact, this applies starting from any bounded (not necessarily summable) initial condition, i.e., for any $u^{0} \neq 0$ in $\ell_{+}^{\infty}(\mathbb{Z})$. (In the trivial case $u^{0}=0$ we have $u^{n}=0$ for all $n$.)

Theorem 6. Let $\mu>0$ and $\alpha$ satisfy (2). Then for each $u^{0} \in \ell_{+}^{\infty}(\mathbb{Z}), u^{0} \neq 0$, there exists $c>0$ such that

$$
\lim _{n \rightarrow \infty} u_{i}^{n}=c \text { for all } i \in \mathbb{Z} .
$$

Let $\pi \in \ell_{+}^{1}(\mathbb{Z})$ be given by

$$
\pi_{i}=\frac{\mu}{\alpha}\left(\frac{\alpha-\mu}{\alpha+\mu}\right)^{|i|}
$$

for each $i \in \mathbb{Z}$; this is a probability measure on $\mathbb{Z}$, i.e., $\sum_{i \in \mathbb{Z}} \pi_{i}=1$. The auxiliary operator $G$ was defined in Section 2 , We have

Proposition 7. For each $u \in \ell_{+}^{\infty}(\mathbb{Z})$

$$
\lim _{n \rightarrow \infty} G_{i}^{(n)}(u)=\pi \cdot u \equiv \sum_{k=-\infty}^{\infty} \pi_{k} u_{k} \text { for all } i \in \mathbb{Z} .
$$


Proof. The linear operator $G$ corresponds to a Markov chain 11 on $\mathbb{Z}$ with transition probabilities given by a stochastic matrix $P$, where $P_{i k}$ is the coefficient of $u_{k}$ in the formula for $G_{i}(u)$ in (4). It is easy to verify that there is a single irreducible component (the whole space $\mathbb{Z}$ when $\alpha>\mu$, and $\{0\}$ when $\alpha=\mu$ ), and that $\pi$ given by (6) has finite mass and satisfies $\pi_{k}=\sum_{i \in \mathbb{Z}} \pi_{i} P_{i k}$ for all $k \in \mathbb{Z}$. Therefore (see Feller [2, Theorem XV.7]), $\pi$ is the unique invariant probability measure of the Markov chain, and $P_{i k}^{n} \rightarrow{ }_{n} \pi_{k}$ for all $i, k \in \mathbb{Z}$, where $P^{n}$ denotes the $n$-th power of the matrix $P$. This implies $G_{i}^{(n)}(u)=\sum_{k} P_{i k}^{n} u_{k} \rightarrow_{n} \sum_{k} \pi_{k} u_{k}$ for any $u \in \ell_{+}^{\infty}(\mathbb{Z})$ (since $\pi \in \ell_{+}^{1}(\mathbb{Z})$ ).

Proposition 7 together with Lemma 2 (iv) readily imply that if $u^{0} \in \ell_{+}^{1}(\mathbb{Z}), u^{0} \neq$ 0 , then the total mass $\left\|u^{n}\right\|$ increases to infinity. We now prove the stronger result of Theorem 6 .

Proof of Theorem 6. Let $M_{n}:=\sup _{i \in \mathbb{Z}} u_{i}^{n}$; the sequence $M_{n}$ is nonincreasing (since each coordinate of $u^{n+1}$ is an average of coordinates of $u^{n}$ ), and so it converges to a limit $M$. Assuming without loss of generality that the 0 -th coordinate $u_{0}^{0}$ of $u^{0}$ is positive yields by Lemma 2(iv) and Proposition 7

$$
M_{n} \geq u_{i}^{n} \geq G_{i}^{(n)}\left(u^{0}\right) \rightarrow_{n} \pi \cdot u^{0} \geq \pi_{0} u_{0}^{0}=\frac{\mu}{\alpha} u_{0}^{0}>0,
$$

hence $M>0$.

We will show that $\lim _{n} u_{i}^{n}=M$ for all $i$. There are three cases.

Case 1: $\alpha=\mu$. Let $\varepsilon>0$, and assume without loss of generality that $u_{0}^{0} \geq M_{0}-\varepsilon$; then (17) implies $\lim _{n} M_{n} \geq u_{0}^{0} \geq M_{0}-\varepsilon$. The sequence $M_{n}$ is nonincreasing, hence $M=\lim _{n} M_{n}=M_{0}$, and using (77) again yields $\lim _{n} u_{i}^{n}=M$ for all $i$.

Case 2: $\alpha>\mu$ and $\alpha+\mu<1 / 2$. For large $n$ the supremum $M_{n}$ stays almost constant (and close to $M$ ), from which we will deduce that there must be an appropriate block of consecutive coordinates that are all close to $M$ (see (91)); we will then apply Proposition 7 (see (10) $)$.

Indeed, let $\varepsilon>0$. Then there exists $K$ such that

$$
\sum_{k=-K}^{K} \pi_{k} \geq 1-\varepsilon,
$$

and there exists $N$ such that

$$
M_{N} \leq M+\varepsilon^{\prime},
$$

where $\varepsilon^{\prime}:=\gamma^{K} \varepsilon$ and $\gamma:=\min \{\alpha-\mu, 1-2 \alpha-2 \mu\}>0$. Let $L:=K+N$ and assume now without loss of generality 2 that $u_{0}^{L} \geq M_{L}-\varepsilon^{\prime}$. Then $u_{0}^{L}=F_{0}^{(K)}\left(u^{N}\right)$ is a convex combination of the coordinates of $u^{N}$ that are at a distance of at most $K$ from 0, i.e.,

$$
u_{0}^{L}=\sum_{k=-K}^{K} \beta_{k} u_{k}^{N},
$$

\footnotetext{
${ }^{1}$ A standard reference for Markov chains is Feller [2] Chapter XV].

${ }^{2}$ Note that $F$ is translation-invariant, and so, instead of centering $G$ at 0 , we could have centered it at any $i_{0}$; this would merely have shifted $\pi$ by $i_{0}$ and left everything unchanged, in particular Lemma 2 and Proposition 7.
} 
where $\sum_{k} \beta_{k}=1$ and $\beta_{k} \geq 0$. While the coefficients $\beta_{k}$ are not fixed (they depend on $u^{N}$ ), they are uniformly bounded away from zero:

$$
\beta_{k} \geq \gamma^{K}>0 \text { for all } k=-K, \ldots, K
$$

(indeed, the nonzero coefficients in $F_{i}(u)-$ of $u_{i-1}, u_{i}$, and $u_{i+1}$ - are all $\geq \gamma$; use induction on $K$ ).

For each $k=-K, \ldots, K$ we have

$$
\begin{aligned}
M-\varepsilon^{\prime} \leq M_{L}-\varepsilon^{\prime} \leq u_{0}^{L} & \leq \beta_{k} u_{k}^{N}+\left(1-\beta_{k}\right)\left(M+\varepsilon^{\prime}\right) \\
& \leq \gamma^{K} u_{k}^{N}+\left(1-\gamma^{K}\right)\left(M+\varepsilon^{\prime}\right)
\end{aligned}
$$

(the last inequality, which is equivalent to $\left(\beta_{k}-\gamma^{K}\right)\left(M+\varepsilon^{\prime}-u_{k}^{N}\right) \geq 0$, follows from (8) and $u_{k}^{N} \leq M_{N} \leq M+\varepsilon^{\prime}$ by our choice of $\left.N\right)$. This implies

$$
u_{k}^{N} \geq M+\varepsilon^{\prime}-\frac{2 \varepsilon^{\prime}}{\gamma^{K}}>M-2 \varepsilon \text { for all } k=-K, \ldots, K
$$

(recall that $\varepsilon^{\prime}=\gamma^{K} \varepsilon$ ).

Finally, applying Lemma 2(iv) and Proposition 7 and recalling the choice of $K$ yields

$$
\begin{aligned}
u_{i}^{n+N} & =F_{i}^{(n)}\left(u^{N}\right) \geq G_{i}^{(n)}\left(u^{N}\right) \\
& \rightarrow_{n} \quad \pi \cdot u^{N} \geq(M-2 \varepsilon) \sum_{k=-K}^{K} \pi_{k} \geq(M-2 \varepsilon)(1-\varepsilon)
\end{aligned}
$$

for all $i$, which completes the proof in this case.

Case 3: $\alpha>\mu$ and $\alpha+\mu=1 / 2$. The proof here is a modification of the argument in the previous case. Since now $1-2 \alpha-2 \mu=0$, some of the coefficients $\beta_{k}$ may vanish: instead of (8) and (9) which hold for all $k=-K, \ldots, K$, we only get similar inequalities for every other $k$ (indeed: the coefficients of $u_{i-1}$ and $u_{i+1}$ in $F_{i}(u)$ are positive, whereas the coefficient of $u_{i}$ may vanish). However, if $y$ is the alternating sequence $y=(\ldots, 1,0,1,0,1,0, \ldots)$, then it is easy to see that $F(y)=(\ldots, 1-\eta, 1,1-\eta, 1,1-\eta, 1, \ldots)$, where $\eta:=2 \alpha-2 \mu<1$, and $F^{(n)}(y)=$ $\left(\ldots, 1,1-\eta^{n}, 1,1-\eta^{n}, 1,1-\eta^{n}, \ldots\right)$ for every $n \geq 1$.

Therefore we proceed as follows: given $\varepsilon>0$, let $R$ be such that $\eta^{R} \leq \varepsilon$, let $K_{0}$ be such that $\sum_{k=-K_{0}}^{K_{0}} \pi_{k} \geq 1-\varepsilon$, and take $K:=K_{0}+R$ and $\gamma:=\alpha-\mu>0$. Continuing as in Case 2 , we now get $\beta_{k} \geq \gamma^{K}>0$, and thus $u_{k}^{N}>M-2 \varepsilon$, for every other $k$ between $-K$ and $K$. Therefore, for all $k=-K_{0}, \ldots, K_{0}$, we have by the monotonicity of $F$ (see Lemma 1 (iv); only the coordinates between $-K$ and $K$ matter here)

$$
u_{k}^{R+N}=F_{k}^{(R)}\left(u^{N}\right) \geq F_{k}^{(R)}((M-2 \varepsilon) y),
$$

where $y$ is the alternating sequence above. The homogeneity of degree 1 of $F$, the computation of $F^{(n)}(y)$ above, and our choice of $R$ imply

$$
u_{k}^{R+N} \geq(M-2 \varepsilon) F_{k}^{(R)}(y) \geq(M-2 \varepsilon)\left(1-\eta^{R}\right) \geq(M-2 \varepsilon)(1-\varepsilon) .
$$

Applying now Proposition 7 as in (10), with $u^{R+N}$ instead of $u^{N}$, yields

$$
\liminf _{n \rightarrow \infty} u_{i}^{n+R+N} \geq(M-2 \varepsilon)(1-\varepsilon)^{2}
$$

for all $i$ (recall the choice of $K_{0}$ ).

The result of Theorem 6 holds in the multi-dimensional case as well. 
Theorem 8. Let $d \geq 1$ be an integer, and let $\mu>0$ and $\alpha$ satisfy (5). Then for each $u^{0} \in \ell_{+}^{\infty}\left(\mathbb{Z}^{d}\right), u^{0} \neq 0$, there exists $c>0$ such that

$$
\lim _{n \rightarrow \infty} u_{i}^{n}=c \text { for all } i \in \mathbb{Z}^{d} .
$$

Indeed, the same arguments apply; the invariant probability measure $\pi$ corresponding to $G$ is now given by

$$
\pi_{i}=\left(\frac{\mu}{\alpha}\right)^{d}\left(\frac{\alpha-\mu}{\alpha+\mu}\right)^{\|i\|}
$$

for each $i \in \mathbb{Z}^{d}$.

\section{REFERENCES}

[1] Ben-Artzi, M., J. Goodman and A. Levy [2000], "Remarks on a Nonlinear Parabolic Equation," Transactions of the American Mathematical Society, 352, 731-751. MR1615935 (2000c:35092)

[2] Feller, W. [1968], An Introduction to Probability Theory and Its Applications, Volume 1, Third Edition, Wiley. MF0228020 (37:3604)

[3] Gilding, B., M. Guedda and R. Kersner [1998], "The Cauchy Problem for the KPZ Equation," prepublication LAMFA 28, Amiens, December 1998.

[4] Laurençot, P. and P. Souplet [2003], "On the Growth of Mass for a Viscous Hamilton-Jacobi Equation," Journal d'Analyse Mathématique, 89, 367-383. MR1981925 (2004c:35188)

Institute of Mathematics, Department of Economics, and Center for the Study of Rationality, Feldman Building, Givat Ram Campus, The Hebrew University of Jerusalem, 91904 Jerusalem, Israel

E-mail address: hart@huji.ac.il

URL: http://www.ma.huji.ac.il/hart

Institute of Mathematics, and Center for the Study of Rationality, The Hebrew University of Jerusalem, 91904 Jerusalem, Israel

E-mail address: weiss@math.huji.ac.il 